\title{
ON DECAY OF SOLUTION TO LINEAR PARABOLIC EQUATION WITH DOUBLE DEGENERACY
}

\section{V.F. VIL'DANOVA}

\begin{abstract}
We obtain the upper bound for the decay rate of the solution to the Dirichlet initial boundary value problem for a linear parabolic second order equation with a double degeneracy $\mu(x) u_{t}=\left(\rho(x) a_{i j}(t, x) u_{x_{i}}\right)_{x_{j}}$ in an unbounded domain. For a wide class of revolution domains we prove a lower bound. We adduce the examples showing that the upper and lower bounds are in some sense sharp.

We prove the unique solvability of the problem in an unbounded domain by Galerkin's approximations method.
\end{abstract}

Keywords: parabolic equation with a double degeneracy, decay rate of a solution, upper bound, existence of a solution.

Mathematics Subject Classification: 35B30, 35B45, 35K10, 35K20, 35K65

\section{INTRODUCTION}

Let $\Omega$ be an unbounded domain in space $\mathbb{R}^{n}, x=\left(x_{1}, x_{2}, \ldots, x_{n}\right) \in \mathbb{R}^{n}, n \geqslant 2$. In a cylindrical domain $D=\{t>0\} \times \Omega$ we consider a linear second order equation

$$
\mu(x) u_{t}=\sum_{i, j=1}^{n}\left(\rho(x) a_{i j}(t, x) u_{x_{i}}\right)_{x_{j}},
$$

where weights $\mu(x)>0$ and $\rho(x)>0$ are measurable functions integrable over each bounded subset of $\Omega: \mu, \rho \in L_{\text {loc }}^{1}(\bar{\Omega})$. For symmetric coefficients $a_{i j}=a_{j i}$ we impose the condition of the uniform ellipticity: there exist positive constants $\gamma, \gamma_{1}$ such that for each vector $y \in \mathbb{R}^{n}$ and almost each $(t, x) \in D$ the identities

$$
\gamma|y|^{2} \leqslant \sum_{i, j=1}^{n} a_{i j}(t, x) y_{i} y_{j} \leqslant \gamma_{1}|y|^{2}
$$

hold true.

On the lateral boundary of cylinder $D$ we impose Dirichlet boundary condition:

$$
\left.u(t, x)\right|_{\Gamma}=0, \quad \Gamma=(0, \infty) \times \partial \Omega .
$$

We shall deal with a generalized solution to problem (1), (3) subject to the initial condition

$$
u(0, x)=\varphi(x) \in L_{2}(\Omega, \mu d x) .
$$

The present work is devoted to the studying the dependence of the decay rate as $t \rightarrow \infty$ of a solution to problem (1), (3), (4) on the geometry of unbounded domain $\Omega$ and behavior of weights $\mu, \rho$ as $x \rightarrow \infty$.

The first studies of the decay rate of the solution to a mixed problem of second order uniformly parabolic equation $(\mu=\rho \equiv 1)$ on the geometry of the unbounded domain were made by A.K. Guschin

V.F. Vil'DANOVA, On DECAY OF SOLUTION TO LINEAR PARABOLIC EQUATION WITH DOUBLE DEGENERACy. (C) VIL'DANOVA V.F. 2016.

The work is supported by the grant for young scientists of the Scientific council of BSPU named after M. Akhmulla.

Submitted October 25, 2015. 
in works [1, 2]. For a wide class of domains there was established the estimate for the solution to the second mixed problem:

$$
|u(t, x)| \leqslant \frac{\|\varphi\|_{L_{1}(\Omega)}}{v(\sqrt{t})}, \quad x \in \Omega,
$$

where $v(r)=\operatorname{mes}\{x \in \Omega:|x|<r\}$. The sharpness of this estimate was proved. In particular, for the solution of Cauchy problem this estimate becomes

$$
|u(t, x)| \leqslant C \frac{\|\varphi\|_{L_{1}\left(\mathbb{R}^{n}\right)}}{(\sqrt{t})^{n}}
$$

More complete studies of the dependence of the behavior at infinity of solution to the second mixed problem on the geometry of the domain and on the initial function were made by A.V. Lezhnev in [3]. V.I. Ushakov [4] obtained the results close to that by A.K. Guschin for the third mixed problem in a non-cylindrical domain. Earlier in work [5] F.Kh. Mukminov proved the estimate for the decay rate of solution to the first mixed problem for a second order uniformly parabolic equation and its sharpness in the class of unboundedly monotonically increasing revolution domains was proved. In work [6] there were obtained sharp estimates for solution to a forth and sixth order parabolic equation with Rickyies condition on the lateral boundary of an unbounded parabolic domain.

We also mention work [7, where the dependence of the solution on the structure of nonlinearities in equations was studied.

A more complete survey of results related to the subject of our work can be found in [6]-[14].

We proceed to formulating our result. We introduce the functions

$$
\lambda(r)=\inf _{g \in C_{0}^{\infty}(\Omega)} F_{r}(g), \quad F_{r}(g)=\frac{\int_{\Omega[r]} \rho(x)|\nabla g|^{2} d x}{\int_{\Omega[r]} \mu g^{2} d x},
$$

where $\Omega[r]=\{x \in \Omega|| x \mid<r\}$;

$$
\widetilde{\lambda}(r)=\inf _{g \in C_{0}^{\infty}(\Omega)} \frac{\int_{S_{r}} \rho(x)|\nabla g|^{2} d S}{\int_{S_{r}} \rho g^{2} d S},
$$

where $S_{r}=\{x \in \Omega|| x \mid=r\}$. It is obvious that function $\lambda(r)$ is bounded on the interval $r>r_{0}$ if set $\Omega\left[r_{0}\right]$ is non-empty.

In the next statement we discuss a generalized solution to problem (see Section 2).

Theorem 1. Let $u(t, x)$ be a solution to problem (1), (3), (4) with the initial function $\varphi$ vanishing as $|x|>R_{0}$. Then there exists a number $\nu_{1}>0$ depending only on $n, \gamma_{1}, R_{0}$ and $T$ and depending also on functions $\lambda, \widetilde{\lambda}$ such that for all $t>T$ the inequality

$$
\int_{\Omega} \mu(x) u^{2}(t, x) d x \leqslant C \exp \left(-\nu_{1} \int_{R_{0}+1}^{r(t)} \sqrt{\tilde{\lambda}(s)} d s\right) \int_{\Omega} \mu(x) \varphi^{2}(x) d x
$$

holds true, where $r=r(t)$ is an arbitrary continuous function satisfying the inequality

$$
t \lambda(r) \geqslant \int_{R_{0}+1}^{r} \sqrt{\tilde{\lambda}(s)} d s
$$

Constant $C$ depends on $\gamma, \gamma_{1}$ and on function $\tilde{\lambda}$.

It is known that in the case of a planar angle $\Omega=\{(r, \psi) \mid r>0,0<\psi<\alpha\}$ as $\mu=\rho \equiv 1$, the decay rate of solution to problem (1), (3), $(4)$ is power: $u(t, x)=O\left(t^{-(\pi / \alpha+1)}\right)$ (see [5]). For such situations (i.e., when the decay of solution is power) estimate (7) provides non-adequate result since the exact value of constant $\nu_{1}$ is not determined (i.e., exponent $t$ is not determined precisely). 
If inequality $\rho(x) \leqslant \mu(x)$ holds true for almost each $x \in \Omega$, we can obtain an estimate slightly weaker than (7) without employing function $\widetilde{\lambda}$ (see Theorem 2 in Section 3).

We note that the theorem remains true if we replace domains $\Omega[r], S_{r}$ by $\Omega(r)=\left\{x \in \Omega \mid x_{1}<r\right\}$ and $S_{r}=\left\{\left(x_{1}, x^{\prime}\right) \in \Omega \mid x_{1}=r\right\}$. At that we assume that domains $\Omega(r)$ are bounded for all $r>0$.

Formally function $r(t)=R_{0}+1$ satisfies the inequality in theorem. But it is clear that inequality (7) becomes stronger if we choose the largest function $r(t) \geqslant R_{0}+1$ among admissible ones. In the case when function $\lambda(r)$ is continuous and positive at least at one point $r \geqslant R_{0}+1$, we define function $r(t)$ as the largest among the roots of the equation $t \lambda(r)=\int_{R_{0}+1}^{r} \sqrt{\tilde{\lambda}(s)} d s$ (for sufficiently large $t$ there exists at least one root). In the end of Section 3 we also provide a condition ensuring the continuity of function $\lambda(r)$. The same approach is applicable under the presence of the estimate $\lambda(r) \geqslant h(r)$ with replacing $\lambda(r)$ by a continuous function $h(r)$. In the simplest case $\rho=\mu=1$ function $h(r)$ can be chosen by employing inequality (5.4) in [21, CH. II, Sect. 5], which in the case mes $\Omega[r] \leqslant(1-\varepsilon)$ mes $B(r)$, where $B(r)$ is the ball of radius $r$, is written as

$$
\int_{\Omega[r]} u^{2}(t, x) d x \leqslant \beta \varepsilon^{-2} r^{2} \int_{\Omega[r]}|\nabla u|^{2}(t, x) d x, \quad \beta>0 .
$$

It implies the inequality $\lambda(r) \geqslant \frac{\varepsilon^{2}}{\beta r^{2}}$. We also observe that inequality $(8)$ applied to the cone with the vertex at point $O$ and spherical basis $S_{r}$ instead of $\Omega[r]$, gives the estimate $\widetilde{\lambda}(r) \geqslant \delta^{2}(r) /\left(\beta r^{2}\right)$, where $1-\delta(r)=\operatorname{mes}_{n-1} S_{r} r^{1-n} / \omega_{n}, \omega_{n}$ is the measure on the unit sphere. In particular, when function $\delta(r)$ decays fast enough, the inequality $\int_{R_{0}+1}^{r} \sqrt{\widetilde{\lambda}(s)} d s<\infty$ is possible and then estimate 7 becomes meaningless.

In Section 4 we also provide examples of functions $r(t)$ for functions $\mu, \rho$ not being equal to 1 . In Section 5 we provide Theorem 2 on the lower bound of the non-negative solution in the case when domain $\Omega$ is a revolution one. By the examples we show that inequality (7) of Theorem 1 is in some sense sharp.

\section{EXISTENCE AND UNIQUENESS OF SOLUTION}

We introduce the following notations: $D_{a}^{b}=(a, b) \times \Omega, D^{T}=D_{0}^{T}, D=D_{0}^{\infty}$,

$$
\|u\|_{D^{T}, \mu}^{2}=\int_{D^{T}} \mu u^{2} d x d t, \quad\|\nabla u\|_{D^{T}, \rho}=\int_{D^{T}} \rho|\nabla u|^{2} d x d t .
$$

On the set of restrictions of functions in $C_{0}^{\infty}\left(D_{-1}^{T}\right)$ on $D^{T}$ we define the norms

$$
\|u\|_{H^{0,1}\left(D^{T}\right)}^{2}=\|u\|_{D^{T}, \mu}^{2}+\|\nabla u\|_{D^{T}, \rho}^{2} ; \quad\|u\|_{H^{1,1}\left(D^{T}\right)}^{2}=\|u\|_{H^{0,1}\left(D^{T}\right)}^{2}+\left\|u_{t}\right\|_{D^{T}, \mu}^{2} .
$$

The completions of these liner normed spaces are denoted by $\stackrel{\circ}{H}^{0,1}\left(D^{T}\right)$ and $\stackrel{\circ}{H}^{1,1}\left(D^{T}\right)$. For the uniqueness of the gradient of the functions in the introduced weighted space we impose the condition in work [20]:

$$
\rho^{-1} \in L_{\mathrm{loc}}^{1}(\Omega)
$$

We defined space $\stackrel{\circ}{H}^{1}(\Omega)$ as the completion of space $C_{0}^{\infty}(\Omega)$ by the norm $\|u\|_{H^{1}(\Omega)}^{2}=\int_{\Omega}\left(\mu u^{2}+\rho|\nabla u|^{2}\right) d x$.

A generalized solution to problem (1), (3), (4) in $D^{T}$ is a function $u(t, x) \in \stackrel{\circ}{H}^{0,1}\left(D^{T}\right)$ satisfying the integral identity

$$
\int_{D^{T}}\left(-\mu u v_{t}+\sum_{i, j=1}^{n} \rho a_{i j}(t, x) u_{x_{i}} v_{x_{j}}\right) d x d t=\int_{\Omega} \mu \varphi(x) v(0, x) d x
$$

for each function $v(t, x) \in \stackrel{\circ}{H}^{1,1}\left(D^{T}\right)$.

Function $u(t, x)$ is a solution to problem (1), (3), (4) in $D$ if for each $T>0$ it is a solution to problem (1), (3), (4) in $D^{T}$. 
The generalized solution of problem (1), (3), (4) in $D^{T}$ exists and is unique. The existence can be proved by Galerkin method (see, for instance, [21]).

We choose a set of linearly independent functions $w_{i}(x) \in C_{0}^{\infty}(\Omega)$ such that their linear span is dense in $\stackrel{\circ}{H}^{1}(\Omega)$. Without loss of generality we can assume that these functions are orthonormalized in $L_{2}(\Omega, \mu d x)$.

We seek Galerkin approximations in the form

$$
u^{l}(t, x)=\sum_{i=1}^{n} C_{i}^{l}(t) w_{i}(x) .
$$

We obtain the equations for the sought coefficients by the restriction

$$
\int_{\Omega}\left(\mu(x) u_{t}^{l} w_{s}+\sum_{i, j=1}^{n} \rho(x) a_{i j}(t, x) u_{x_{i}}^{l}\left(w_{s}\right)_{x_{j}} d x=0, \quad s=\overline{1, l} .\right.
$$

Thanks to the orthonormaliaty of functions $w_{i}$, conditions (11) lead us to the system of ordinary differential equations

$$
\left(C_{i}^{l}\right)^{\prime}+\sum_{j=1}^{n} b_{i j}(t) C_{j}^{l}=0, \quad i=\overline{1, n} .
$$

We choose the initial conditions for system of differential equations 12 as

$$
C_{i}^{l}(0)=\left(\varphi, w_{i}\right)
$$

Conditions 12, 13 determine the unique set of functions $C_{i}^{l}(t)$.

Let us prove the boundedness of set $u^{l}$ of Galerkin approximations in space $\stackrel{\circ}{H}^{0,1}\left(D^{T}\right)$. We multiply identities (11) by $C_{s}^{l}$ and sum they up. We obtain

$$
\int_{\Omega}\left(\mu u_{t}^{l} u^{l}+\sum_{i, j=1}^{n} \rho a_{i j}(t, x) u_{x_{i}}^{l} u_{x_{j}}^{l}\right) d x d t=0 .
$$

Integrating (14) over $t \in(0, T)$ and employing condition (2), we get

$$
\frac{1}{2} \int_{\Omega} \mu(x)\left[\left(u^{l}(t, x)\right)^{2}-\left(u^{l}(0, x)\right)^{2}\right] d x+\gamma \int_{D^{T}} \rho(x)\left|\nabla u^{l}\right|^{2} d x d t \leqslant 0 .
$$

It is obvious that

$$
\left\|u^{l}(0, x)\right\|_{L_{2}(\Omega, \mu d x)}^{2}=\sum_{i=1}^{l}\left(\varphi, w_{i}\right)^{2} .
$$

Then (15) can be rewritten as

$$
\left.\int_{\Omega} \mu(x) u^{l}(t, x)\right)^{2} d x+2 \gamma \int_{D^{T}} \rho(x)\left|\nabla u^{l}\right|^{2} d x d t \leqslant\|\varphi\|_{D^{T}, \mu}^{2} .
$$

It implies the boundedness of set $u^{l}$ in subspace $\stackrel{\circ}{H}^{0,1}\left(D^{T}\right)$. This is why we can choose a subsequence weakly converging in this space to some function $u \in \stackrel{\circ}{H}^{0,1}\left(D^{T}\right)$. In order to avoid cumbersome superscripts, we assume that the sequence weakly converges itself.

We multiply (11) by function $d_{s}(t) \in C_{0}^{\infty}(-1, T)$ and integrate over $t \in(0, T)$. By denoting $v=d_{s} w_{s}$, integrating by parts and passing to the limit as $l \rightarrow \infty$, we obtain

$$
\int_{D^{T}}\left(-\mu u(v)_{t}+\sum_{i, j=1}^{n} \rho a_{i j}(t, x) u_{x_{i}}(v)_{x_{j}} d x d t\right)=\int_{\Omega} \mu \varphi(x) v(0, x) d x .
$$

We note that (17) is true not only for functions $v=d_{s} w_{s}$, but also for the sums of such functions. It remains to mention that by functions $v^{m}=\sum_{s=1}^{m} d_{s} w_{s}$, we can approximate each function $w$ in $C_{0}^{\infty}\left(D_{-1}^{T}\right)$ in the norm of space $\stackrel{\circ}{H}^{1,1}\left(D^{T}\right)$.

Let us show the uniqueness of solution to problem (1), (3), (4). 
By $v_{h}(t, x)$ we denote the Steklov average of function $v(t, x)$ :

$$
v_{h}(t, x)=\frac{1}{h} \int_{t}^{t+h} v(\tau, x) d \tau,
$$

which possesses the following properties:

1) $\left(v, u_{-h}\right)=\left(v_{h}, u\right)_{L_{2}\left(\mathbb{R}^{n+1}, \mu d x d t\right)}$, where $(v, u)_{L_{2}\left(\mathbb{R}^{n+1}, \mu d x d t\right)}=\int_{\mathbb{R}^{n+1}} \mu v u d x d t$,

2) if $v \in \stackrel{\circ}{H}^{0,1}\left(D_{0}^{T}\right)$, then $\left(v_{h}\right)_{x_{i}}=\left(v_{x_{i}}\right)_{h}$,

3) if $v, v_{t} \in L_{2}\left(\mathbb{R}^{n+1}, \mu d x d t\right)$, then $\left(v_{t}\right)_{h}=\left(v_{h}\right)_{t}$,

4) if $v \in L_{2}\left(D^{T}, \mu d x d t\right)$, then for each $\delta>0$ the convergence $v_{h} \rightarrow v$ is true in $L_{2}\left(D^{T-\delta}, \mu d x d t\right)$ as $h \rightarrow 0(h<\delta)$.

We substitute test function $v_{-h}$ into integral identity $(9)$, where $v$ belongs to space $C_{0}^{\infty}\left(D_{0}^{T-\delta}\right)$. It is possible since $v_{-h} \in C_{0}^{\infty}\left(D_{0}^{T}\right)$ as $0<h<\delta$. Employing the properties of Steklov average, we get

$$
\int_{D^{T}}\left[\mu\left(u_{h}\right)_{t} v+\rho \sum_{i, j=1}^{n}\left(a_{i j} u_{x_{i}}\right)_{h} v_{x_{j}}\right] d x d t=0 .
$$

By passing to the limit we prove that the latter relation is true not only for $v \in C_{0}^{\infty}\left(D_{0}^{T-\delta}\right)$, but also for $v \in \stackrel{\circ}{H^{0,1}}\left(D_{0}^{T-\delta}\right)$.

We note that identities 18 are of the form

$$
\int_{D^{T}} \mu\left(u_{h}\right)_{t} v d x d t=l_{h}(v)
$$

where $l_{h}(v)$ is a linear functional in space $\stackrel{\circ}{H}^{0,1}\left(D_{0}^{T-\delta}\right)$.

Let us prove the uniform boundedness of linear functional $l_{h}(v)$ as $|h|<\delta_{0}$ in the unit ball of space $\stackrel{\circ}{H}^{0,1}\left(D_{0}^{T-\delta}\right)$.

We consider $l_{h}(v)$ and in view of the uniform ellipticity we have

$$
\begin{aligned}
\left|l_{h}(v)\right| & =\left|\int_{D^{T-\delta}} \rho \sum_{i, j=1}^{n}\left(a_{i j} u_{x_{i}}\right)_{h} v_{x_{j}} d x d t\right| \leqslant \int_{D^{T-\delta}}\left(\frac{\gamma_{1}}{h} \int_{t}^{t+h} \rho|\nabla u(\tau, x)| d \tau\right)|\nabla v(t, x)| d x d t \\
& \leqslant \int_{D^{T-\delta}} \gamma_{1} \rho\left(\frac{1}{h^{2}}\left(\int_{t}^{t+h}|\nabla u(\tau, x)| d \tau\right)^{2}+|\nabla v(t, x)|^{2}\right) d x d t .
\end{aligned}
$$

Thus, we obtain that $\left|l_{h}(v)\right| \leqslant C$. The boundedness of linear functional $l_{h}(v)$ is proved.

We substitute function $v=\left(u_{h_{1}}-u_{h_{2}}\right) \chi\left(t_{1}, t_{2}\right) \in \stackrel{\circ}{H}^{0,1}\left(D_{0}^{T-\delta}\right)$ into identities $\left[19 h_{h_{1}}-19 h_{h_{2}}\right.$, where $\chi\left(t_{1}, t_{2}\right)$ is the characteristic function of the interval $\left(t_{1}, t_{2}\right)$. We obtain

$$
\left|\int_{t_{1}}^{t_{2}} \int_{\Omega} \mu\left(\left(u_{h_{1}}\right)_{t}-\left(u_{h_{2}}\right)_{t}\right)\left(u_{h_{1}}-u_{h_{2}}\right) d x d t\right|=\left|\left(l_{h_{1}}-l_{h_{2}}\right)\left(\chi\left(u_{h_{1}}-u_{h_{2}}\right)\right)\right| \leqslant C\left\|\left(u_{h_{1}}-u_{h_{2}}\right)\right\|_{H^{0,1}} \leqslant \varepsilon .
$$

The latter inequality for sufficiently small $h_{1}, h_{2}$ is implied by the convergence $u_{h} \rightarrow u$ in space $\stackrel{\circ}{H}^{0,1}\left(D_{0}^{T-\delta}\right)$. After integration in $t$ we obtain

$$
\int_{\Omega} \mu\left(u_{h_{1}}-u_{h_{2}}\right)^{2}\left(t_{1}, x\right) d x \leqslant \int_{\Omega} \mu\left(u_{h_{1}}-u_{h_{2}}\right)^{2}\left(t_{2}, x\right) d x+2 \varepsilon .
$$

We integrate this inequality in $t_{2} \in\left[t_{1}, T-\delta\right]$ :

$$
\left(T-\delta-t_{1}\right) \int_{\Omega} \mu\left(u_{h_{1}}-u_{h_{2}}\right)^{2}\left(t_{1}, x\right) d x \leqslant \mu\left\|\left(u_{h_{1}}-u_{h_{2}}\right)\right\|_{L_{2}\left(D^{T-\delta}, \mu d x\right)}^{2}+2 \varepsilon\left(T-\delta-t_{1}\right) .
$$


Since $u_{h} \rightarrow u$ in $L_{2}\left(D^{T-\delta}, \mu d x\right)$, as $t_{1}<T-2 \delta$ we have the inequality

$$
\int_{\Omega} \mu\left(u_{h_{1}}-u_{h_{2}}\right)^{2}\left(t_{1}, x\right) d x \leqslant \frac{\varepsilon_{1}}{\delta}+2 \varepsilon .
$$

It yields the uniform fundamentality in $t_{1}$ of the family of functions $u_{h}\left(t_{1}, x\right)$ in $L_{2}(\Omega, \mu d x)$. This is why $u_{h}(t, x) \rightrightarrows u(t, x)$ in $L_{2}(\Omega, \mu d x)$ as $h \rightarrow 0$ uniformly in $t \in[0, T-2 \delta]$ and the limiting function is continuous in $t$ in the norm of $L_{2}(\Omega, \mu d x)$. We substitute function $v=u_{h} \chi(0, t)$ into (18):

$$
\int_{D_{0}^{t}}\left(\mu\left(u_{h}\right)_{t} u_{h}+\rho \sum_{i, j=1}^{n}\left(a_{i j} u_{x_{i}}\right)_{h}\left(u_{h}\right)_{x_{j}}\right) d x d t=0 .
$$

Integrating the first term in $t$ and passing to the limit as $h \rightarrow 0$, we obtain

$$
\frac{1}{2} \int_{\Omega} \mu u^{2}(t, x) d x+\int_{D_{0}^{t}} \rho \sum_{i, j=1}^{n} a_{i j} u_{x_{i}} u_{x_{j}} d x d t=\frac{1}{2} \int_{\Omega} \mu u^{2}(0, x) d x .
$$

If we prove that $u(0, x)=\varphi(x)$, the latter relation coincides with $(9)$. In order to do it, we substitute a continuous test function $v(t, x)=\eta\left(\frac{t}{\varepsilon}\right) \psi(x)$ into identity (9), where $\eta(t)=1-t$ as $t \in[0,1]$ and $\eta(t)$ is constant in other intervals $(-\infty, 0],[1, \infty)$. Since $v_{t}=-\frac{1}{\varepsilon} \psi(x)$, identity (9) becomes

$$
\int_{0}^{\varepsilon} \int_{\Omega} \frac{1}{\varepsilon} \mu(x) \psi(x) u(t, x) d t d x+l^{\varepsilon}(\psi)=\int_{\Omega} \mu(x) \varphi(x) \psi(x) d x,
$$

where linear functional $l^{\varepsilon}(\psi)$ tends to zero as $\varepsilon \rightarrow 0$. Passing to the limit as $\varepsilon \rightarrow 0$, we obtain

$$
\int_{\Omega} \mu(x) \psi(x) u(0, x) d x=\int_{\Omega} \mu(x) \varphi(x) \psi(x) d x
$$

for each $\psi \in C_{0}^{\infty}(\Omega)$. It proves the intitial condition $u(0, x)=\varphi(x)$.

\section{UPPER BOUND FOR SOLUTION}

We first establish two estimates characterising the decay of solution to problem (1), (3), (4) as $|x| \rightarrow \infty$.

Proposition 1. Let $u(t, x)$ be a solution to problem (1), (3), (4) with initial function $\varphi$ vanishing outside the ball of radius $R_{0}$. Suppose that the inequality

$$
\rho(x) \leqslant C \mu(x), \quad C>0, \quad x \in \Omega,
$$

holds true. Then for all $t>0, r \geqslant R_{0}$ the inequality

$$
\int_{\Omega \backslash \Omega[r]} \mu u^{2}(t, x) d x \leqslant e \exp \left(-\widetilde{C} t^{-1}\left(r-R_{0}\right)^{2}\right) \int_{\Omega} \mu(x) \varphi^{2}(x) d x
$$

holds true, where $\widetilde{C}$ is a constant depending on $\gamma$ and $\gamma_{1}$.

Proof. Let $\xi(\tau, r, \varrho)$ be a continuous nonnegative function vanishing as $\tau \leqslant r$ and being one as $\tau \geqslant$ $r+\varrho$. In the remaining interval it is linear: $\frac{\partial \xi}{\partial \tau}=\frac{1}{\varrho}$. We substitute a test function $v=\eta(x ; r, \varrho) u_{h}$, $\eta(x)=\xi^{2}(|x|, r, \varrho)$ into identity 18 to obtain

$$
\int_{D^{T}}\left[\frac{1}{2} \mu\left(u_{h}^{2} \eta\right)_{t}+\sum_{i, j=1}^{n} \rho\left(a_{i j} u_{x_{i}}\right)_{h}\left(\eta u_{h}\right)_{x_{j}}\right] d x d t=0 .
$$

We passing to the limit as $h \rightarrow 0$ in identity 23 :

$$
\int_{\Omega} \mu\left(u^{2}(T, x)-\varphi^{2}(x)\right) \eta d x+2 \int_{D^{T}} \sum_{i, j=1}^{n} \rho a_{i j} u_{x_{i}}(\eta u)_{x_{j}} d x d t=0 .
$$


By the condition $\operatorname{supp} \varphi \subset \Omega\left[R_{0}\right]$, for each $r \geqslant R_{0}$ and $\varrho>0$ we obtain easily the inequality

$$
\begin{aligned}
\int_{\Omega} \mu \eta u^{2}(T, x) d x+2 \int_{D^{T}} \rho \sum_{i, j=1}^{n} \eta a_{i j} u_{x_{i}} u_{x_{j}} d x d t & \leqslant-2 \int_{D^{T}} \rho \sum_{i, j=1}^{n} a_{i j} u_{x_{i}} u \frac{\partial \eta}{\partial x_{j}} d x d t \\
& \leqslant 2 \int_{D^{T}} \rho \gamma_{1}|u \nabla u \nabla \eta| d x d t .
\end{aligned}
$$

Rewriting the latter, we have

$$
\int_{\Omega} \mu \eta u^{2}(T, x) d x+\int_{D^{T}} \gamma \rho \eta|\nabla u|^{2} d x d t \leqslant 2 \int_{D^{T}} \rho \gamma_{1}|u \nabla u \nabla \eta| d x d t .
$$

Employing the structure of function $\eta$, we obtain easily the inequality

$$
\int_{\Omega \backslash \Omega[r+\varrho]} \mu u^{2}(t, x) d x+\int_{0}^{t} \int_{\Omega \backslash \Omega[r+\varrho]} \rho \gamma|\nabla u|^{2} d x d t \leqslant \frac{C}{\varrho^{2}} \int_{0}^{t} \int_{\Omega[r+\varrho] \backslash \Omega[r]} \rho u^{2} d x d t .
$$

We introduce the notation

$$
H_{r}(t)=\int_{\Omega \backslash \Omega[r]} \mu u^{2}(x, t) d x+\int_{0}^{t} \int_{\Omega \backslash \Omega[r]} \rho \gamma|\nabla u|^{2} d x d t
$$

and employ condition (21) to establish that

$$
H_{r+\varrho}(t) \leqslant \frac{C}{\varrho^{2}} \int_{0}^{t} H_{r}(\tau) d \tau
$$

We apply inequality (25) inductively to sequence $r_{i}, i=0,1,2, \ldots k, r_{i+1}=r_{i}+\varrho, r_{0}=R_{0}$. Since (20) implies the inequality

$$
H_{r}(t) \leqslant A=\int_{\Omega} \mu(x) \varphi^{2}(x) d x, \quad r>0, \quad t>0,
$$

we get

$$
H_{R_{0}+\varrho}(t)=\frac{A C t}{\varrho^{2}}
$$

Let us establish the inequality

$$
H_{r_{k}}(t) \leqslant \frac{A C^{k} t^{k}}{\varrho^{2 k} k !}
$$

by induction in $k$.

Indeed,

$$
H_{r_{k}+\varrho}(t) \leqslant \frac{C}{\varrho^{2}} \int_{0}^{t} H_{r_{k}}(\tau) d \tau \leqslant \frac{C}{\varrho^{2}} \int_{0}^{t} \frac{A C^{k} \tau^{k}}{\varrho^{2 k} k !} d \tau=\frac{A C^{k+1} t^{k+1}}{\varrho^{2(k+1)}(k+1) !}
$$

that completes the induction. Employing Stirling's inequality, by (27) we obtain easy that

$$
H_{r_{k}}(t) \leqslant \frac{A C^{k} e^{k} t^{k}}{\sqrt{2 \pi k} \varrho^{2 k} k^{k}} \leqslant A \exp \left(-k \ln \frac{\varrho^{2} k}{C e t}\right) .
$$

We choose $k$ equal to the integer part of the number $\frac{\left(r-R_{0}\right)^{2}}{C e^{2} t}$. If $k=0$, then $\frac{\left(r-R_{0}\right)^{2}}{C e^{2} t}<1$ and $H_{r}(t) \leqslant A=e A e^{-1}$ that implies inequality 222. If $k \geqslant 1$, then $k \geqslant \frac{\left(r-R_{0}\right)^{2}}{2 C e^{2} t}$. Now we let $\varrho=$ $\left(r-R_{0}\right) / k$. Then $r_{k}=r$ and $\varrho^{2} k=\frac{\left(r-R_{0}\right)^{2}}{k} \geqslant C e^{2} t$. Therefore, $\frac{\varrho^{2} k}{C e t} \geqslant 1$. Hence it follows from 28) that $H_{r}(t)=H_{r_{k}}(t) \leqslant A e^{-k}$. It leads us to inequality $(22)$. 
Proposition 2. Let $u(t, x)$ be the solution to problem (1), (3), (4) with an initial function $\varphi$ vanishing outside a ball of radius $R_{0}$. Then for all $t>0, r \geqslant R_{0}+1$ the inequality

$$
\int_{\Omega \backslash \Omega[r]} \mu u^{2}(t, x) d x \leqslant C \exp \left(-2 \nu \int_{R_{0}+1}^{r} \sqrt{\tilde{\lambda}(s)} d s\right) \int_{\Omega} \mu(x) \varphi^{2}(x) d x
$$

holds true, where $C, \nu$ are constants depending on $\gamma$ and $\gamma_{1}$, while $C$ depends also on function $\widetilde{\lambda}$.

Proof. Let $\xi(\tau, r)$ be a continuous nonnegative function vanishing as $\tau \leqslant R_{0}$, being linear as $R_{0}<$ $\tau<R_{0}+1$ and equalling one as $\tau \geqslant r$. In the remaining interval it satisfies the condition $\frac{\partial \xi}{\partial \tau}=\nu \sqrt{\tilde{\lambda} \xi}$, where number $\nu$ will be fixed later.

It is easy to see that $\xi_{\tau}=\xi\left(R_{0}+1, r\right)$ as $\tau \in\left(R_{0}, R_{0}+1\right)$, where

$$
\xi\left(R_{0}+1, r\right)=\exp \left(-\nu \int_{R_{0}+1}^{r} \sqrt{\tilde{\lambda}(s)} d s\right) .
$$

We substitute a test function $v=\eta(x ; r) u_{h}, \eta(x ; r)=\xi^{2}(|x|, r)$, into identity (18) to obtain

$$
\int_{D^{T}}\left[\frac{1}{2} \mu\left(u_{h}^{2} \eta\right)_{t}+\sum_{i, j=1}^{n} \rho\left(a_{i j} u_{x_{i}}\right)_{h}\left(\eta u_{h}\right)_{x_{j}}\right] d x d t=0 .
$$

Passing to the limit as $h \rightarrow 0$ in identity (30), we get

$$
\int_{\Omega} \mu\left(u^{2}(T, x)-\varphi^{2}(x)\right) \eta d x+2 \int_{D^{T}} \sum_{i, j=1}^{n} \rho a_{i j} u_{x_{i}}(\eta u)_{x_{j}} d x d t=0 .
$$

It implies easily the inequality

$$
\int_{\Omega} \mu \eta u^{2}(T, x) d x+2 \int_{D^{T}} \rho \sum_{i, j=1}^{n} \eta a_{i j} u_{x_{i}} u_{x_{j}} d x d t \leqslant 2 \int_{D^{T}} \rho \gamma_{1}|u \nabla u \nabla \eta| d x d t .
$$

Transforming the latter, we obtain

$$
\int_{\Omega} \xi^{2} \mu u^{2} d x+\int_{D^{T}} \gamma \rho \xi^{2}|\nabla u|^{2} d x d t \leqslant \int_{D^{T}} \rho \gamma_{1}\left(\varepsilon \xi^{2}|\nabla u|^{2}+\frac{u^{2} \xi^{\prime 2}}{\varepsilon} d x\right) d t .
$$

Taking $\varepsilon=\frac{\gamma}{2 \gamma_{1}}$, we obtain the inequality

$$
\begin{aligned}
\int_{\Omega} \xi^{2} \mu u^{2} d x & +\frac{\gamma}{2} \int_{D^{T}} \rho \xi^{2}|\nabla u|^{2} d x d t \\
& \leqslant \frac{2 \gamma_{1}^{2}}{\gamma}\left(\int_{0}^{T} \int_{\Omega[r] \backslash \Omega\left[R_{0}+1\right]} \nu^{2} \rho u^{2} \xi^{2} \widetilde{\lambda} d x d t+\int_{0}^{T} \int_{\Omega\left[R_{0}+1\right] \backslash \Omega\left[R_{0}\right]} \rho u^{2} \xi^{2}\left(R_{0}+1\right) d x d t\right) .
\end{aligned}
$$

Employing the definition of function $\xi$, we rewrite the latter terms:

$$
\begin{aligned}
\int_{\Omega[r] \backslash \Omega\left[R_{0}+1\right]} \nu^{2} \rho u^{2} \xi^{2} \widetilde{\lambda} d x & =\int_{R_{0}+1}^{r} \nu^{2} \xi^{2}(\tau) \widetilde{\lambda}(\tau) d \tau \int_{S_{\tau}} \rho u^{2} d S \\
& \leqslant \int_{R_{0}+1}^{r} \nu^{2} \xi^{2}(\tau) d \tau \int_{S_{\tau}} \rho|\nabla u|^{2} d S=\nu^{2} \int_{\Omega[r] \backslash \Omega\left[R_{0}+1\right]} \rho \xi^{2}|\nabla u|^{2} d x .
\end{aligned}
$$


In the same way,

$$
\int_{\Omega\left[R_{0}+1\right] \backslash \Omega\left[R_{0}\right]} \rho u^{2} d x \leqslant \frac{1}{\inf _{\left[R_{0}, R_{0}+1\right]} \tilde{\lambda}(\tau)} \int_{\Omega\left[R_{0}+1\right] \backslash \Omega\left[R_{0}\right]} \rho|\nabla u|^{2} d x .
$$

We let $\nu=\frac{\gamma}{2 \gamma_{1}}$. Substituting $(33)$ and $(34)$ into $(32)$ and estimating the right hand side in (34) by 20 , we obtain inequality $29, C=2 \gamma_{1}^{2} /\left(\gamma_{\left[R_{0}, R_{0}+1\right]} \lambda(\tau)\right)$.

Theorem 2. Let $u(t, x)$ be a solution to problem (1), (3), (4) with an initial function $\varphi$ vanishing as $|x|>R_{0}$ and let inequality (21) hold true. Then there exists a constant $\nu_{2}>0$ depending only on $n, \gamma_{1}, R_{0}$ such that for all $t>0$ the inequality

$$
\int_{\Omega} \mu(x) u^{2}(t, x) d x \leqslant C \exp \left(-\nu_{2} t \lambda(r(t))\right) \int_{\Omega} \mu(x) \varphi^{2}(x) d x
$$

holds true, where $r=r(t)$ is an arbitrary function satisfying the inequality $t \lambda(r) \leqslant t^{-1}\left(r-R_{0}\right)^{2}$. Constant $C$ depends only on $\gamma, \gamma_{1}$ and $n$.

Proof of Theorems 1, 2. Let $T>0$ be an arbitrary number. We introduce the notation

$$
\varepsilon=\sup _{t \in[0, T]} \int_{\Omega \backslash \Omega[r]} \mu u^{2}(t, x) d x .
$$

The inequality

$$
\int_{\Omega} \mu u^{2}(t, x) d x \leqslant \varepsilon+\int_{\Omega[r]} \mu u^{2}(t, x) d x
$$

holds true. Since for almost each $t \in(0, T)$ function $u(t, x)$ is an element of space $\stackrel{\circ}{H}^{1}(\Omega)$, by (5) we obtain

$$
\int_{\Omega} \mu u^{2}(t, x) d x \leqslant \varepsilon+\lambda^{-1}(r) \int_{\Omega} \rho|\nabla u|^{2} d x .
$$

By means of relation

$$
\frac{d}{d t} \int_{\Omega} \mu u^{2}(t, x) d x \leqslant-\gamma \int_{\Omega} \rho|\nabla u|^{2} d x
$$

implied by 20 , for the function $E(t)=\int_{\Omega} \mu u^{2}(t, x) d x$ we get the inequality

$$
\gamma(E(t)-\varepsilon) \lambda(r) \leqslant-\frac{d}{d t} E(t) .
$$

Solving this inequality, we find

$$
E(T)-\varepsilon \leqslant e^{-T \lambda(r) \gamma} E(0)
$$

To prove Theorem 2, we make use of estimate $(22)$ :

$$
\varepsilon \leqslant e \exp \left(-\widetilde{C} T^{-1}\left(r-R_{0}\right)^{2}\right) \int_{\Omega} \mu(x) \varphi^{2}(x) d x .
$$

Then

$$
E(T) \leqslant E(0)\left(e \exp \left(-\widetilde{C} T^{-1}\left(r-R_{0}\right)^{2}\right)+e^{-T \lambda(r) \gamma}\right) .
$$

The latter inequality is valid for all $r \geqslant R_{0}$. It is natural to find the infimum of the right hand side in $r$. But since we can find constructively the point of the infimum, we can take value $r(T)>R_{0}$ (as small as possible) to satisfy the inequality

$$
T^{-1}\left(r-R_{0}\right)^{2} \geqslant T \lambda(r) .
$$

The possibility of such choice for $r(T)$ follows from the boundedness of function $\lambda(r)$. Substituting $r=r(T)$ into (39), we arrive at the estimate in Theorem 2 . 
To prove Theorem 1, we employ estimate (29):

$$
\varepsilon \leqslant C \exp \left(-2 \nu \int_{R_{0}+1}^{r} \sqrt{\tilde{\lambda}(s)} d s\right) \int_{\Omega} \mu \varphi^{2}(x) d x .
$$

We choose $r=r(T)$ (as large as possible) so that

$$
T \lambda(r) \geqslant \int_{R_{0}+1}^{r} \sqrt{\tilde{\lambda}(s)} d s .
$$

Then (38) implies inequality (7) of Theorem 1.

Let us show that function $\lambda(r)$ is continuous in a rather general situation. We call domain $\Omega$ regular if there exists a family of diffeomorphisms $\varphi_{r_{1}, r_{2}}: \Omega\left[r_{1}\right] \rightarrow \Omega\left[r_{2}\right], 0<r_{1}<r_{2}$, such that $\varphi_{r_{1}, r_{2}}(x) \rightarrow i d$ in $C^{1}\left(\Omega\left[r_{1}\right]\right)$ both as $r_{1} \rightarrow r_{2}$ and as $r_{2} \rightarrow r_{1}$.

Let us show for a regular domain function $\lambda(r)$ is continuous/ For each $\varepsilon>0, r>0$ there exists a function $g_{r} \in C_{0}^{1}(\Omega)$ (depending on $\varepsilon$ ) such that $F_{r}\left(g_{r}\right)<\lambda(r)+\varepsilon$. It is obvious that $\lambda\left(r_{1}\right) \leqslant F_{r_{1}}\left(g_{r_{2}}\right)$. This is why

$$
\lim \sup _{r_{1} \rightarrow r_{2}} \lambda\left(r_{1}\right) \leqslant \lambda\left(r_{2}\right)+\varepsilon .
$$

Then $F_{r_{2}}\left(g_{r_{1}}\left(\varphi_{r_{1}, r_{2}}(x)\right)\right) \geqslant \lambda\left(r_{2}\right)$. Therefore,

$$
\begin{aligned}
\lambda\left(r_{2}\right) & \leqslant \lim \inf _{r_{1} \rightarrow r_{2}} F_{r_{2}}\left(g_{r_{1}}\left(\varphi_{r_{1}, r_{2}}(x)\right)\right)=\lim \inf _{r_{1} \rightarrow r_{2}} F_{r_{1}}\left(g_{r_{1}}\left(\varphi_{r_{1}, r_{2}}(x)\right)\right) \\
& =\lim \inf _{r_{1} \rightarrow r_{2}} F_{r_{1}}\left(g_{r_{1}}(x)\right) \leqslant \varepsilon+\lim \inf _{r_{1} \rightarrow r_{2}} \lambda\left(r_{1}\right) .
\end{aligned}
$$

Thanks to the arbitrariness of $\varepsilon>0$, the obtained relations yield the left continuity of function $\lambda(r)$. The right continuity can be proven in the same way.

\section{EXAmples}

We restrict ourselves by constructing examples in the case $n=2$, while similar examples can be easily adapted for a multi-dimensional situation for the revolution domain

$$
\Omega_{f}=\left\{\left(x_{1}, x^{\prime}\right)\left|x_{1}>0 ;\right| x^{\prime} \mid<f\left(x_{1}\right)\right\}
$$

defined by a positive continuous function $f\left(x_{1}\right), f\left(x_{1}\right) \geqslant 1, x_{1}>0$. We shall obtain some estimates for functions $\lambda, \tilde{\lambda}$ in the case of planar domain $\Omega_{f}$.

For the simplicity we shall refer to the version of Theorem 1, when domains $\Omega[r], S_{r}$ are replaced by $\Omega(r)=\left\{x \in \Omega \mid x_{1}<r\right\}$ and $S_{r}=\left\{\left(x_{1}, x^{\prime}\right) \in \Omega \mid x_{1}=r\right\}$.

Let us establish an analogue of Steklov-Fridrichs inequality with weights. Let $g(s) \in C^{1}[0, r]$ and $g(0)=0$. Squaring the identity

$$
g(s)=g(s)-g(0)=\int_{0}^{s} g^{\prime}(t) d t
$$

it is easy to obtain

$$
g^{2}(s) \leqslant \int_{0}^{r} \rho^{-1}(t) d t \int_{0}^{r} \rho(t)\left(g^{\prime}(t)\right)^{2} d t
$$

Then we multiply by $\mu(s)$ and integrate in $s$ :

$$
\int_{0}^{r} \mu(s) g^{2}(s) d s \leqslant \int_{0}^{r} \mu(s) d s \int_{0}^{r} \rho^{-1}(t) d t \int_{0}^{r} \rho(t)\left(g^{\prime}(t)\right)^{2} d t .
$$


Assume that $g\left(x_{1}, x_{2}\right) \in C_{0}^{\infty}(\Omega)$. Then we have

$$
\int_{0}^{f\left(x_{1}\right)} \mu(x) g^{2}(x) d x_{2} \leqslant \int_{0}^{f\left(x_{1}\right)} \mu(x) d x_{2} \int_{0}^{f\left(x_{1}\right)} \rho^{-1}(x) d x_{2} \int_{0}^{f\left(x_{1}\right)} \rho(x)\left(g_{x_{2}}^{\prime}(x)\right)^{2} d x_{2} .
$$

We introduce the notation $\Lambda(r)=\sup _{0 \leqslant x_{1} \leqslant r} M\left(x_{1}\right)$, where

$$
M\left(x_{1}\right)=\int_{0}^{f\left(x_{1}\right)} \mu(x) d x_{2} \int_{0}^{f\left(x_{1}\right)} \rho^{-1}(x) d x_{2} .
$$

Then

$$
\int_{0}^{f\left(x_{1}\right)} \mu(x) g^{2}(x) d x_{2} \leqslant \Lambda(r) \int_{0}^{f\left(x_{1}\right)} \rho(x)\left(g_{x_{2}}^{\prime}(x)\right)^{2} d x_{2} .
$$

or, integrating in $x_{1}$, we obtain

$$
\int_{\Omega[r]} \mu(x) g^{2}(x) d x \leqslant \Lambda(r) \int_{\Omega[r]} \rho(x)\left(g_{x_{2}}^{\prime}(x)\right)^{2} d x .
$$

As $\mu(x)$ and $\rho(x)$ we consider the functions

$$
\begin{aligned}
& \rho\left(x_{1}, x_{2}\right)= \begin{cases}\widetilde{\rho}\left(x_{1}\right)\left(f\left(x_{1}\right)-\left|x_{2}\right|\right)^{\alpha}, & \left|x_{2}\right| \in\left[f\left(x_{1}\right)-1, f\left(x_{1}\right)\right] \mid, \\
\widetilde{\rho}\left(x_{1}\right), & \left|x_{2}\right|<f\left(x_{1}\right)-1,\end{cases} \\
& \mu\left(x_{1}, x_{2}\right)= \begin{cases}\widetilde{\mu}\left(x_{1}\right)\left(f\left(x_{1}\right)-\left|x_{2}\right|\right)^{\beta}, & \left|x_{2}\right| \in\left[f\left(x_{1}\right)-1, f\left(x_{1}\right)\right] \mid, \\
\widetilde{\mu}\left(x_{1}\right), & \left|x_{2}\right|<f\left(x_{1}\right)-1,\end{cases}
\end{aligned}
$$

where $|\alpha|<1, \beta>-1$. We shall define function $\widetilde{\mu}\left(x_{1}\right), \widetilde{\rho}\left(x_{1}\right)$ later. For simplicity, we shall assume that $f(r) \geqslant \frac{|\alpha|}{1-|\alpha|}$ and $f(r) \geqslant \frac{-\beta}{1+\beta}$ as $r \geqslant R_{0}$.

Calculating $M\left(x_{1}\right)$ as $\mu=\rho$, by (41) we find that

$$
\widetilde{\lambda}(r) \geqslant\left[\left(f(r)-\frac{\alpha}{1+\alpha}\right)\left(f(r)+\frac{\alpha}{1-\alpha}\right)\right]^{-1} \geqslant \frac{1}{2 f^{2}(r)}
$$

as $r \geqslant R_{0}$. Substituting this estimate into $(29)$, we obtain

$$
\int_{\Omega \backslash \Omega(r)} \mu u^{2}(t, x) d x \leqslant C \exp \left(-2 \nu \int_{R_{0}+1}^{r} \frac{d s}{f(s)}\right) \int_{\Omega} \mu(x) \varphi^{2}(x) d x .
$$

It is easy to see that

$$
\begin{aligned}
\Lambda(r) & =\sup _{0 \leqslant x_{1} \leqslant r} \frac{\widetilde{\mu}\left(x_{1}\right)}{\widetilde{\rho}\left(x_{1}\right)}\left(f\left(x_{1}\right)-\frac{\beta}{1+\beta}\right)\left(f\left(x_{1}\right)+\frac{\alpha}{1-\alpha}\right) \\
& \leqslant \max \left(\sup _{0 \leqslant x_{1} \leqslant r} 4 \frac{\widetilde{\mu}\left(x_{1}\right)}{\widetilde{\rho}\left(x_{1}\right)} f^{2}\left(x_{1}\right), \sup _{0 \leqslant x_{1} \leqslant R_{0}} 4 \frac{\widetilde{\mu}\left(x_{1}\right)}{\widetilde{\rho}\left(x_{1}\right)} \frac{|\beta|}{1+\beta} \frac{|\alpha|}{1-\alpha}\right) .
\end{aligned}
$$

For the sake of simplicity we assume that function $\frac{\widetilde{\mu}\left(x_{1}\right)}{\widetilde{\rho}\left(x_{1}\right)} f^{2}\left(x_{1}\right)$ increases and

$$
\frac{\widetilde{\mu}\left(R_{0}\right)}{\widetilde{\rho}\left(R_{0}\right)} f^{2}\left(R_{0}\right) \geqslant \sup _{0 \leqslant x_{1} \leqslant R_{0}} \frac{\widetilde{\mu}\left(x_{1}\right)}{\widetilde{\rho}\left(x_{1}\right)} \frac{|\beta|}{1+\beta} \frac{|\alpha|}{1-\alpha} .
$$

Due to 42 we have $\lambda(r) \geqslant \Lambda^{-1}(r)$ and this is why

$$
\lambda(r) \geqslant \frac{\widetilde{\rho}(r)}{4 \widetilde{\mu}(r) f^{2}(r)} .
$$


Roughing a little bit the estimate in Theorem 1 (see its proof), we can choose function $r(t)$ satisfying the inequality $\frac{t \widetilde{\rho}(r)}{\widetilde{\mu}(r) f^{2}(r)} \geqslant \int_{R_{0}+1}^{r} \frac{d s}{f(s)}$. Then estimate 7 becomes

$$
\int_{\Omega} \mu(x) u^{2}(t, x) d x \leqslant C \exp \left(-\nu_{2} \int_{R_{0}+1}^{r(t)} \frac{d s}{f(s)}\right) \int_{\Omega} \mu(x) \varphi^{2}(x) d x .
$$

In particular, if $f(s)=s^{p}, p \in(0,1)$, then

$$
\int_{R_{0}+1}^{r(t)} \frac{d s}{f(s)} \leqslant \frac{r^{1-p}}{1-p}
$$

Suppose for simplicity that

$$
\frac{\widetilde{\rho}}{\widetilde{\mu}}=\frac{r^{q}}{1-p}, \quad q<1-p,
$$

then the inequality determining $r(t)$ casts into the form $t \geqslant r^{1-p-q}$ and we can choose $r(t)=t^{(1-p-q)^{-1}}$. In this case estimate 45 becomes

$$
\int_{\Omega} \mu(x) u^{2}(t, x) d x \leqslant C_{1} \exp \left(-C_{2} t^{\frac{1-p}{1-p-q}}\right) \int_{\Omega} \mu(x) \varphi^{2}(x) d x .
$$

We observe that in the multi-dimensional case function $f(s)=s^{p}$ generates the revolution paraboloid and all the above arguments remain true with appropriately changed constants.

In the case $f(s)=s$ we have the interior of an angle in the plane (or of a cone in the multidimensional case). Then

$$
\int_{R_{0}+1}^{r(t)} \frac{d s}{f(s)} \leqslant \ln r
$$

As an example we choose functions $\widetilde{\rho}, \widetilde{\mu}$ so that $\frac{\widetilde{\rho}}{\widetilde{\mu}}=\frac{\ln r}{r^{q}}, q>0$. Then the inequality determining $r(t)$ becomes $t \geqslant r^{q}$. We choose $r(t)=t^{1 / q}$. Then estimate 45 casts into the form

$$
\int_{\Omega} \mu(x) u^{2}(t, x) d x \leqslant C_{3} \exp \left(-C_{4} \ln t\right) \int_{\Omega} \mu(x) \varphi^{2}(x) d x .
$$

\section{LOWER BOUND}

We recall Harnack inequality established by J.A. Moser for a uniformly parabolic equation [23]

$$
u_{t}=\sum_{i, j=1}^{n}\left(a_{i j}(t, x) u_{x_{i}}\right)_{x_{j}} .
$$

We formulate it in a convenient for us form: for a nonnegative in a cylinder $Q=\left(0,9 C_{1} \rho^{2}\right] \times B(2 \rho, \mathbf{w}) \subset$ $\mathbb{R}^{n+1}, C_{1}>1$ solution to equation 46 the inequality

$$
\max _{Q^{-}} u(\tau, x) \leqslant H \min _{Q^{+}} u(\tau, x),
$$

holds true, where $Q^{-}=\left[\rho^{2}, 2 \rho^{2}\right] \times B(\rho, \mathbf{w}), Q^{+}=\left[8 C_{1} \rho^{2}, 9 C_{1} \rho^{2}\right] \times B(\rho, \mathbf{w}), B(\rho, \mathbf{w})$ is the ball of radius $\rho$ centered at point $\mathbf{w} \in \Omega$, and constant $H \geqslant 1$ depends only on $n, C_{1}$ and the parabolicity constants of the equation.

We recall the notion of $A_{2}$-weight introduced by Muckenhoupt. This is a measurable function $\vartheta(x)$ : $\mathbb{R}^{n} \rightarrow \mathbb{R}_{+}$satisfying the inequality

$$
\int_{K} \vartheta(x) d x \times \int_{K} \frac{1}{\vartheta(x)} d x<C_{0}|K|^{2}
$$


for each cube $K \subset \mathbb{R}^{n}$. It was proven in work 24 that if the identity $\rho=\mu=\vartheta$ holds in $Q$, where $\vartheta$ is some $A_{2}$-weight, then for each nonnegative in $Q$ solution of equation (1) Harnack inequality holds true. At that, constant $H$ depends only on $C_{0}, C_{1}, n, \gamma$ and $\gamma_{1}$. Let us show that we can get rid of the assumption $\rho=\mu$ if $\mu=\vartheta$ and the inequalities

$$
C_{1}^{-1} \leqslant \frac{\rho(x) \mu(\mathbf{w})}{\mu(x) \rho(\mathbf{w})} \leqslant C_{1}, \quad x \in B(2 \rho, \mathbf{w}),
$$

hold true. By the change $\tau=\frac{\rho(\mathbf{w})}{\mu(\mathbf{w})} t$ we obtain the equation

$$
\operatorname{div}(\rho(x) a(t, x) \nabla u)=\mu(x) u_{t}=\frac{\mu(x) \rho(\mathbf{w})}{\mu(\mathbf{w})} u_{\tau}
$$

or as $x \in B(2 \rho, \mathbf{w})$ :

$$
\operatorname{div}\left(\vartheta(x) \frac{\rho(x) \mu(\mathbf{w})}{\mu(x) \rho(\mathbf{w})} a(\tau, x) \nabla u\right)=\vartheta(x) u_{\tau} .
$$

The latter equation in $Q$ is of the form $\sqrt{1}$ with $\rho=\mu=\vartheta$ and $\widetilde{a}=\frac{\rho(x) \mu(\mathbf{w})}{\mu(x) \rho(\mathbf{w})} a$. If variables $(\tau, x) \in Q$, then $(t, x) \in \widetilde{Q}=\left(0,9 C_{1} \rho^{2} \frac{\mu(\mathbf{w})}{\rho(\mathbf{w})}\right] \times B(2 \rho, \mathbf{w})$. Then $Q^{-} \rightarrow \widetilde{Q}^{-} Q^{+} \rightarrow \widetilde{Q}^{+}$change obviously. For these new cylinders, Harnack inequality is still true.

Thus, if in the neighbourhood of each point $\mathbf{w} \in \Omega$ the inequality 47 holds true and in this neighborhood function $\mu(x)$ coincides with some weight $\vartheta$ (depending on point $\mathbf{w}$ ), then a non-negative solution to equation (1) is either positive everywhere in $\Omega$ or vanishes identically. This can be proven by the standard technique if the radius of the neighbourhood depends continuously on the point.

In what follows we shall consider a positive solution to equation (1).

Theorem 3. Assume that $s>p f(s), p \in(0,1)$ as $s \geqslant z_{0}, \Omega_{f}$ is a revolution domain and weight $\mu(x)$ coincides with some $A_{2}$-weight $\vartheta$ in $\Omega_{p f} \cap\left\{x_{1}>z_{0}\right\}$. Assume that the inequalities

$$
\frac{f\left(x_{1}^{\prime}\right)}{f\left(x_{1}^{\prime \prime}\right)} \leqslant 2, \quad \frac{\rho\left(x^{\prime}\right) \mu\left(x^{\prime \prime}\right)}{\mu\left(x^{\prime}\right) \rho\left(x^{\prime \prime}\right)} \leqslant C_{1}
$$

hold true for all $x^{\prime}, x^{\prime \prime} \in \Omega_{p f}$ such that $x_{1}^{\prime}, x_{1}^{\prime \prime} \in[s-p f(s), s+p f(s)]$ and all $s \geqslant z_{0}$. Then for a positive solution of equation (1) the inequality

$$
\min _{x \in B\left(r^{\prime}, \mathbf{w}\right)} u(t, x) \geqslant u\left(t_{1},\left(z_{0}, 0\right)\right) \exp \left(-C_{2} \int_{z_{0}}^{\widetilde{r}(t)} \frac{d s}{f(s)}\right)
$$

holds true, where $B\left(2 r^{\prime}, \mathbf{w}\right)$ is some ball inscribed in $\Omega_{p f} \cap\left\{z_{0}<x_{1}<\widetilde{r}(t)\right\}, t_{1}>0$ is some fixed number, $\widetilde{r}(t), t \geqslant t_{1}$, is introduced as the smallest $r$ satisfying the inequality

$$
\int_{z_{0}}^{r} \frac{d s}{f(s)} \geqslant t L(r), \quad L(r)=\inf _{\left[z_{0}, r\right]} \frac{4 \rho(z, 0)}{\mu(z, 0) p f^{2}(z)},
$$

while constant $C_{2}$ depends only on $p, C_{0}, C_{1}, n, \gamma, \gamma_{1}$.

Proof. Let $y_{0}=z_{0}$ and $r \geqslant z_{0}$ be an arbitrary number. We construct a sequence of balls with radii $r_{i}, i=1,2, \ldots$, and touching points $\mathbf{v}_{i}=\left(y_{i-1}+2 r_{i}, 0\right)$ such that the double ball $B\left(2 r_{i}, \mathbf{w}_{i}\right)$, where $\mathbf{w}_{i}=\left(z_{i}, 0\right), z_{i}=y_{i-1}+r_{i}$, touches the set $\partial \Omega_{p f}$ from inside. We note that $r_{i+1} \leqslant 3 r_{i}$ since otherwise $B\left(2 r_{i}, \mathbf{w}_{i}\right) \subset B\left(2 r_{i+1}, \mathbf{w}_{i+1}\right)$, i.e., ball $B\left(2 r_{i}, \mathbf{w}_{i}\right)$ does not touch the boundary of $\Omega_{p f}$.

We denote $\mu_{i}=\mu\left(\mathbf{w}_{i}\right), \rho_{i}=\rho\left(\mathbf{w}_{i}\right), t_{1}=r_{1}^{2} \frac{\mu_{1}}{\rho_{1}} ; t_{i+1}=t_{i}+\left(9 C_{1}-1\right) \frac{\mu_{i}}{\rho_{i}} r_{i}^{2}$.

If for some $i$ the inequality $r_{i} \leqslant r_{i+1}$ holds true, then as $s=z_{i+1}$ we have $s-z_{i} \leqslant 2 r_{i+1} \leqslant p f(s)$ and by $(48)$ we obtain the inequality

$$
\frac{\mu_{i+1} \rho_{i}}{\rho_{i+1} \mu_{i}} \leqslant C_{1}
$$


If $r_{i}>r_{i+1}$, then letting $s=z_{i}, z_{i+1}-s<2 r_{i}<p f(s)$, by (48) we again obtain (49). Moreover, as $s=z_{i}$, by (48) we get also an analogue of inequality (47):

and inequality

$$
C_{1}^{-1} \leqslant \frac{\rho(x) \mu\left(w_{i}\right)}{\mu(x) \rho\left(w_{i}\right)} \leqslant C_{1}, x \in B\left(2 \rho_{i}, w_{i}\right)
$$

$$
\frac{f\left(x_{1}^{\prime}\right)}{f\left(x_{1}^{\prime \prime}\right)} \leqslant 2, \forall x^{\prime}, x^{\prime \prime} \in\left[s-2 r_{i}, s+2 r_{i}\right]
$$

Consider the cylinders

$$
\begin{aligned}
& \widetilde{Q}_{i}=\left[t_{i}-\frac{\mu_{i}}{\rho_{i}} r_{i}^{2}, t_{i}+\left(9 C_{1}-1\right) \frac{\mu_{i}}{\rho_{i}} r_{i}^{2}\right] \times B\left(2 r_{i}, \mathbf{w}_{i}\right), \\
& \widetilde{Q}_{i}^{-}=\left[t_{i}, t_{i}+\frac{\mu_{i}}{\rho_{i}} r_{i}^{2}\right] \times B\left(r_{i}, \mathbf{w}_{i}\right), \\
& \widetilde{Q}_{i}^{+}=\left[t_{i}+\left(8 C_{1}-1\right) \frac{\mu_{i}}{\rho_{i}} r_{i}^{2}, t_{i}+\left(9 C_{1}-1\right) \frac{\mu_{i}}{\rho_{i}} r_{i}^{2}\right] \times B\left(r_{i}, \mathbf{w}_{i}\right) .
\end{aligned}
$$

Let us show that if $t_{i+1} \leqslant T$, then $\widetilde{Q}_{i} \subset(0, T] \times \Omega_{p f}$. It is sufficient to establish that $t_{i} \geqslant \frac{\mu_{i}}{\rho_{i}} r_{i}^{2}$. The first step of induction is made. Then due to 49

$$
t_{i+1}=t_{i}+\left(9 C_{1}-1\right) \frac{\mu_{i}}{\rho_{i}} r_{i}^{2} \geqslant 9 C_{1} \frac{\mu_{i}}{\rho_{i}} r_{i}^{2} \geqslant \frac{\mu_{i+1}}{\rho_{i+1}} r_{i+1}^{2}
$$

that completes the induction.

Let $k$ be the first index such that $y_{k+1} \geqslant r$ or $t_{k+1} \geqslant T$. Then by Harnack inequality

$$
u\left(t_{1},\left(y_{0}, 0\right)\right) \leqslant H u\left(t_{2}, \mathbf{v}_{1}\right) \leqslant \ldots \leqslant H^{k} u\left(t_{k+1}, \mathbf{v}_{k}\right) .
$$

It yields $u\left(t_{k+1}, \mathbf{v}_{k}\right) \geqslant H^{-k} C_{3}$. Let us estimate number $k$ from above. Let $s_{i}$ be the abscissa for one of the points, where the ball $B\left(2 r_{i}, \mathbf{w}_{i}\right)$ touches the boundary of domain $\Omega_{p f}$. It is clear that $\left|z_{i}-s_{i}\right| \leqslant 2 r_{i}$, $p f\left(s_{i}\right) \leqslant 2 r_{i}$ and this is why due to $[50), f(s) / 2 \leqslant f\left(s_{i}\right)$ as $s \in\left[y_{i-1}, y_{i}\right]$, and $r_{i} \geqslant p f\left(z_{i}\right) / 4$. Then

$$
k=\sum_{i=1}^{k} \frac{y_{i}-y_{i-1}}{2 r_{i}} \leqslant \sum_{i=1}^{k} \frac{y_{i}-y_{i-1}}{p f\left(s_{i}\right)} \leqslant \sum_{i=1}^{k} \int_{y_{i-1}}^{y_{i}} \frac{2 d s}{p f(s)} \leqslant \int_{y_{0}}^{r} \frac{2 d s}{p f(s)} .
$$

Let $\frac{\mu_{m}}{\rho_{m}} r_{m}^{2}=\max _{j \leqslant k} \frac{\mu_{j}}{\rho_{j}} r_{j}^{2} \geqslant \max _{z \in\left[z_{0}, r\right]} \frac{\mu(z, 0)}{64 C_{1} \rho(z, 0)}(p f(z))^{2}$. The latter inequality follows from 448. For the indices $i=m+1, m+2, \ldots$ we replace the balls $B\left(2 r_{i}, \mathbf{w}_{i}\right)$ by the balls $B\left(2 r_{m}, \mathbf{w}_{m}\right)$. Cylinders $\widetilde{Q}_{i}, i=m+1, m+2, \ldots$ change appropriately. Since each cylinder increases $t_{i}$ by $\left(9 C_{i}-1\right) \frac{\mu_{m}}{\rho_{m}} r_{m}^{2}$, then to reach value $t$ we need at most

$$
N=\left[\frac{t \rho_{m}}{\left(9 C_{1}-1\right) \mu_{m} r_{m}^{2}}\right] \leqslant 2 t L(r) / p
$$

cylinders. Thus, we obtain the estimate

$$
\min _{x \in B\left(r_{m}, \mathbf{w}_{m}\right)} u(t, x) \geqslant H^{-(k+N)} C_{3} \geqslant \exp \left(-\left(\int_{z_{0}}^{r} \frac{2 d s}{p f(s)}+2 t L(r) / p\right) \ln H\right),
$$

that implies the statement of the theorem.

We apply inequality (51) to the example in Section 4 to obtain

$$
L(r)=\inf _{\left[z_{0}, r\right]} \frac{4 \widetilde{\rho}(z)}{\widetilde{\mu}(z) p f^{2}(z)}=\frac{4 \widetilde{\rho}(r)}{\widetilde{\mu}(r) p f^{2}(r)} \leqslant 16 \lambda(r) / p .
$$

Employing also inequality (43), we have

$$
\int_{\Omega(r)} \mu(x) u^{2}(t, x) d x \geqslant \pi r_{m}^{2} \min _{x \in B\left(r_{m}, v_{m}\right)} \mu(x) C_{3} \exp \left(-\frac{8}{p^{2}} \ln H\left(\int_{z_{0}}^{r} p \sqrt{\widetilde{\lambda}}(s) d s\right)+8 t \lambda(r)\right) .
$$


Now the choice of $r=r(t)$ as in Introduction (under the assumption $\mathrm{f}$ continuity of function $\lambda(r)$ ):

$$
t \lambda(r)=\int_{R_{0}+1}^{r} \sqrt{\tilde{\lambda}(s)} d s,
$$

justifies in some sense the sharpness of upper estimate (7) if the factor at the exponential in the latter inequality is not too small.

The author express a sincere gratitude F.Kh. Mukminov for the discussion of the results and useful remarks.

\section{BIBLIOGRAPHY}

1. A. K. Gushchin, The estimates of the solutions of boundary value problems for a second order parabolic equation // Trudy Matem. Inst. AN SSSR Stekl. 126, 5-45 (1973). [Proc. Steklov Inst. Math. 126, 1-46 (1973).]

2. A. K. Gushchin. Stabilization of the solutions of the second boundary value problem for a second order parabolic equation // Matem. Sborn. 101(143):4(12), 459-499 (1976). [Math. USSR-Sb. 30:4, 403-440 (1976).]

3. A.V. Lezhnëv. On the behavior, for large time values, of nonnegative solutions of the second mixed problem for a parabolic equation // Matem. Sborn. 129:2,186-200 (1986). [ Math. USSR -Sb. 57:1, 195-209 (1987).]

4. V.I. Ušakov. Stabilization of solutions of the third mixed problem for a second order parabolic equation in a noncylindrical domain // Matem. Sborn. 111(153):1, 95-115 (1980). [Math. USSRSb. 39:1, 87-105 (1981).]

5. F.H. Mukminov. Stabilization of solutions of the first mixed problem for a parabolic equation of second order // Matem. Sborn. 111(153):4, 503-521 (1980). [Math. USSR-Sb. 39:4, 449-467 (1981).]

6. F.Kh. Mukminov, I.M. Bikkulov. Stabilization of the norm of the solution of a mixed problem in an unbounded domain for parabolic equations of orders 4 and 6 // Matem. Sborn. 195:3, 115-142 (2004). [Sb. Math. 195:3, 413-440 (2004).]

7. V.F. Akulov, A.E. Shishkov. On asymptotic properties of solutions of mixed problems for quasilinear parabolic equations in unbounded domains // Matem. Sborn. 182:8, 1200-1210 (1991). [Sb. Math. 73:2, 557-567 (1992).]

8. A.K. Gushchin. On the uniform stabilization of solutions of the second mixed problem for a parabolic equation // Matem. Sborn. 119(161):4(12), 451-508 (1982). [Math. USSR Sb. 47:2, 439-498 (1984).]

9. A. K. Gushchin. Some properties of a generalized solution of the second boundary-value problem for a parabolic equation // Matem. Sborn. 97(139:2(6), 242-261 (1975). [Math. USSR-Sb. 26:2 225-244 (1975).]

10. V.N. Denisov. Stabilization of a solution of a Cauchy problem for the heat-conduction equation // Differ. Uravn. 24:2, 288-299 (1988). [Differ. Equat. 24:2, 219-227 (1988).]

11. V.V. Zhikov. On the stabilization of solutions of parabolic equations // Matem. Sborn. 104(146):4(12), 597-616 (1977). [Math. USSR-Sb. 33:4, 519-537 (1977).]

12. L.M. Kozhevnikova, F.Kh. Mukminov. Estimates of the stabilization rate as $t \rightarrow \infty$ of solutions of the first mixed problem for a quasilinear system of second-order parabolic equations // Matem. Sborn. 191:2, 91-131 (2000). [Sb. Math. 191:2, 235-273 (2000).]

13. L.M. Kozhevnikova. On uniqueness classes of solutions of the first mixed problem for a quasilinear second-order parabolic system in an unbounded domain // Izv. RAN. Ser. Matem. 65:3, 51-66 (2001). [Izv. Math. 65:3, 469-484 (2001).]

14. L.M. Kozhevnikova. Stabilization of a solution of the first mixed problem for a quasi-elliptic evolution equation // Matem. Sborn. 196:7, 67-100 (2005). [Sb. Math. 196:7, 999-1032 (2005).]

15. F.Kh. Mukminov. Stabilization of solution to Dirichet initial boundary value problem for NavierStokes equations. Habilitaion thesis. Steklov Mathematical Institute, Moscow (1994). (in Russian). 
16. F.Kh. Mukminov. Decrease with time of the norm of a solution of a mixed problem for a high-order parabolic equation // Differ. Uravn. 23:10, 1772-1780 (1987). [Differ. Equat. 23:10, 1215-1220 (1987).]

17. A.F. Tedeev. Stabilization of solutions of a first mixed boundary-value problem for a high-order quasilinear parabolic equation // Differ. Uravn. 25:3, 491-498 (1989). [Differ. Equat. 25:3, 346-352 (1989).]

18. A.F. Tedeev. Estimates of the rate of stabilization for $t \rightarrow \infty$ of the solution of the second mixed problem for a second order quasilinear parabolic equation // Differ. Uravn. [Differ. Equat. 27:10, 1274-1283 (1991).]

19. V.I. Ushakov. The behavior for $t \rightarrow \infty$ of solutions of the third mixed problem for second-order parabolic equations // Differ. Uravn. 15, 310-320 (1979). [Differ. Equations 15, 212-219 (1979).]

20. V.V. Zhikov. Weighted Sobolev spaces // Matem. Sborn. 189:8, 27-58 (1998). [Sb. Math. 189:8, 1139-1170 (1998).

21. O.A. Ladyzhenskaya, V.A. Solonnikov, N.N. Ural'tseva. Linear and quasi-linear equations of parabolic type. Nauka, Moscow (1967). [Trans. Math. Mon. 23. Amer. Math. Soc., Providence, RI (1968).]

22. N.V. Krylov, M.V. Safonov. A certain property of solutions of parabolic equations with measurable coefficients // Izv. AN SSSR. Ser. Matem. 44:1, 161-175 (1980). [Math. USSR-Izv. 16:1, 151-164 (1981).]

23. J.A. Moser. Harnack inequality for parabolic differential equations // Comm. Pure Appl. Math. 17:1, 101-134 (1964).

24. F. Chiarenza, R. Serapioni. A remark on a Harnack inequality for degenerate parabolic equations // Rend. Sem. Mat. Univ. Padova. 73, 179-190 (1985).

Venera Fidarisovna Vil'danova

Bashkir State Pedagogical University named after M.Akhmulla,

October rev. str., $3 \mathrm{a}$

450000, Ufa, Russia

E-mail: gilvenera@mail.ru 